\title{
Neuronal polarity in CNS development
}

\author{
David J. Solecki, Eve-Ellen Govek, Toshifumi Tomoda, ${ }^{1}$ and Mary E. Hatten ${ }^{2}$ \\ Laboratory of Developmental Neurobiology, The Rockefeller University, New York, New York 10021, USA
}

The diversity of neuronal morphologies and the complexity of synaptic connections in the mammalian brain provide striking examples of cell polarity. Over the past decade, the identification of the PAR (for partitioningdefective) proteins, their function in polarity in the Caenorhabditis elegans zygote, and the conservation of polarity proteins related to the PAR polarity complex in Drosophila and vertebrates, kindled intense interest in polarity pathways. Although the existence of a conserved polarity protein complex does not prove that these proteins function the same way in different systems, the emergence of an evolutionarily conserved mechanism that regulates cell polarity provides an exciting opportunity to define the role of polarity proteins in the generation of the diverse array of cell types and patterns of connections in the developing mammalian brain. This review addresses emerging genetic, molecular genetic, biochemical, and cell biological approaches and mechanisms that control neuronal polarity, focusing on recent studies using the neonatal cerebellum and hippocampus as model systems.

The Caenorhabditis elegans embryo is a model system for studying cell polarity. Three PAR (for partitioningdefective) polarity proteins localize in the anterior cortex of the one-cell embryo: PAR-3, which contains three PDZ domains; PAR-6, which contains a single PDZ domain and a G-protein-binding, CRIB-like domain; and PKC-3, an atypical protein kinase $\mathrm{C}$ (abbreviated as aPKC, PKC $)$ ). In Drosophila, homologs of the C. elegans PAR proteins are essential for the emergence of cellularity, and for asymmetric division of neuroblasts (Doe and Bowerman 2001; Pellettieri and Seydoux 2002; Gao and Macara 2004; Macara 2004). Mammalian homologs of PAR-3, PAR-6, and PKC-3 (Par3, Par6, and aPKC/PKC $\zeta)$ form a protein complex (mPar6 $\alpha)$ that also localizes in the apical domain of the cell (Kim 2000; Wodarz et al. 2000; Doe and Bowerman 2001).

Many features of intercellular junctions are conserved

[Keywords: Polarity; CNS development; neurogenesis; migration; axon outgrowth; synaptogenesis]

${ }^{1}$ Present address: City of Hope National Medical Center, Beckman Research Institute, Division of Neurosciences, Duarte, CA 91010, USA.

${ }^{2}$ Corresponding author.

E-MAIL hatten@mail.rockefeller.edu; FAX (212) 327-7145.

Article is online at http://www.genesdev.org/cgi/doi/10.1101/gad.1462506. across species from yeast to vertebrates. The apical junction in yeast shares common features with the Drosophila zonula adherens (ZA) junction and ZA junctions of mammalian epithelia, including a band of actin, the transmembrane adhesion proteins, and associated cytoplasmic proteins such as $\beta$-catenin. Polarity proteins that establish the polarity of intercellular junctions of Drosophila epithelia are related to vertebrate polarity proteins that generate intercellular junctions in increasingly complex, multilayered vertebrate epithelia (Fig. 1). In mammalian epithelia, the first step in epithelial cell polarization is the formation of adhesion junctions between cells. Two classes of transmembrane adhesion proteins, cadherins and nectins, initiate adhesions between epithelial cells. Aggregation of adhesion sites leads to the recruitment of cytoskeletal proteins; the association of the intracellular domains of cadherins and nectin activates the Cdc42 and Rac GTPase pathways. After the adherens junction forms, a second adhesion site forms at the apical side of the cell, which becomes a tight junction. In polarized mammalian epithelial cells, the conserved polarity proteins PAR-3/ASIP, PAR-6, and aPKC localize to the tight junction. Recent studies suggest that Par3 and Par6/aPKC form complexes with other polarity proteins as well (Gao and Macara 2004; Macara 2004; Chen and Macara 2005), and contribute to Rac activation downstream of Cdc42 during the polarization of mammalian CNS neurons (Nishimura et al. 2005).

\section{Polarity proteins in neurogenesis}

In the vertebrate nervous system, the neural tube forms from the neural plate just prior to the onset of neurogenesis. Over the past several years, genetic analyses of murine neural tube formation development suggest that mammalian genes related to Drosophila Planar Cell Polarity $(\mathrm{PCP})$ pathway (frizzled $[f z]$, disheveled [dsh], strabismus [stbm/van gogh \{vangl\}], flamingo [fmi], and prickle [pk]) function in the formation of the mammalian neural tube, and in the development of several regions of the mammalian CNS (Torban et al. 2004). Mutations in one of the two forms of the Stbm/Vangl polarity protein Vangl2 gene cause the neural tube defect (craniorachischisis) characteristic of the Looptail (Lp) mutant mouse.

In mammals, the neuroepithelium lining the third ventricle is the germinal zone (VZ) of the neocortex, one 


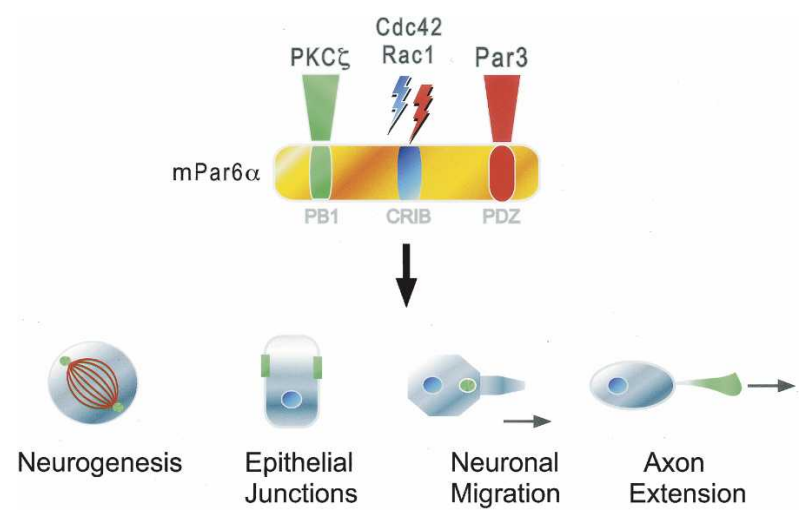

Figure 1. The PAR-3-PAR-6-aPKC protein complex. All three proteins are required for establishing anterior/posterior cell polarity in the zygote of invertebrates. The complex localizes cellular components needed for the asymmetric division of Drosophila, and the formation of epithelial junctions. The polarity complex, termed the mPar6 $\alpha$ signaling complex in vertebrates, exhibits polarized localization in a variety of cell types and organisms. Activated Cdc42 and Rac1 (lightning bolt icons) bind to mammalian PAR-6 (Par6) via a CRIB domain; Par3 (red) binds to Par6 via its PDZ domain and sequesters the activity of the aPKC/PKC $\zeta$ (labeled green). The polarity complex localizes the mitotic spindle during asymmetric divisions of neural progenitors (lower left) and localizes adhesion proteins that orient the mitotic spindle of immature, epithelial cells (second from left). (Second from right) In the mammalian CNS, mPar6 $\alpha$ signaling controls glial-guided migration by setting the tempo of movement of the migrating neuron along the glial fiber. In addition, polarity proteins localize the centrosome (green) just prior to axon outgrowth (left panel) and modulate cytoskeletal dynamics at the growth cone (lower right). Although it is unlikely that polarity protein assemblies function in the same molecular pathways in different cell types or stages of development or parallel steps in different species, evidence is accumulating to suggest that the Par proteins and their signaling pathways are central to a core polarity mechanism(s) conserved in metazoan cells.

of the best-studied cases of CNS neurogenesis. As cells in the cortical VZ divide, a specialized form of polarity emerges that involves "to and fro nuclear migration" (Sauer and Chittenden 1959), with cell divisions restricted to cells with nuclei targeted to the apical pole of the cell. During prophase, the cells release intercellular epithelial contacts and fall to the apical surface of the epithelium (ventricular surface) where they divide. This mode of neurogenesis occurs throughout the developing CNS.

During neuroblast division, the microtubule-organizing center (MTOC) nucleates astral microtubules required for spindle orientation. Cdc42, aPKC, and dynein/ dynactin activity control spindle orientation and govern symmetric versus asymmetric patterns of neuroblast division (Yu et al. 2006). The mechanism of "checkpoint control" is critical for CNS neurogenesis as this step governs assembly of the mitotic spindle, and subsequent segregation of chromosomes into daughter cells. In fungi, proteins homologous to the Lis protein, dynactin and the dynein motor complex, control the polarity of nuclear division. In Drosophila, Lis1/dynactin complexes physically associate and colocalize on centrosomes, spindle microtubules, and kinetochores to regulate multiple, independent functions in mitotic cells, including spindle formation and cell cycle checkpoint release (Siller et al. 2005). Although a series of studies of Lis in developing murine brain initially focused on directed cell migrations (for review, see Hatten 2005), Vallee and Kriegstein (Tsai et al. 2005) showed that a loss of Lis protein results in abnormal mitotic spindles during precursor cell proliferation, a pronounced decrease in interkinetic nuclear migration, and defective neuronal polarity in differentiating neurons prior to directed migrations on glial fibers (Faulkner et al. 2000; O'Connell and Wang 2000). Thus, recent studies on Lis/dynein function in mammalian CNS suggest that the human brain malformation Lissencephaly (for review, see Gleeson and Walsh 2000; Gleeson 2001) relates to defects in spindle orientation, centrosomes, and kinechore functions during CNS neurogenesis.

Neurogenesis in the mammalian neocortex is complicated by the fact that a second germinal zone forms above the primary neuroepithelium, and the fact that in the later cell cycles, radial glial cells, which also function as a guidance system for directed neuronal migration (for review, see Hatten 2002) act as "mother cells," generating neurons via asymmetric division (Anthony et al. 2004; Noctor et al. 2004). Studies by Zhong (Petersen et al. 2002, 2004) provide evidence that the polarity protein Numb is expressed in early phases of cortical histogenesis. Temple and colleagues (Sun et al. 2005) showed that Numb colocalizes with EGFR by a process that is actin dependent. To determine whether the roles of Numb and Numb-like (Numbl) proteins change as neurogenesis progresses, Zhong and colleagues (Petersen et al. 2004) conditionally ablated both genes in the neocortex at later phases of neurogenesis. The loss of Numb and Numbl causes premature progenitor cell depletion and, consequently, malformation of the neocortex and hippocampus. They proposed that Numb-mediated asymmetric cell divisions represent a general mechanism for cell cycle control mechanisms that act to allocate cell fate in the developing mammalian brain (Petersen et al. 2004).

An especially interesting aspect of cortical polarity in CNS neurogenesis is the fact that proteins related to the Drosophila polarity proteins Notch and Numb function both as polarity proteins and as mitogens. Mouse mutants lacking the mammalian form of $\mathrm{Lgl}$, a protein linked to Numb distribution in Drosophila (Langevin et al. 2005), develop structures similar to primitive neuroectodermal tumors and severe hydrocephalus, and die neonatally (Klezovitch et al. 2004). The vast majority of Lgl1 $1^{-/-}$progenitors fail to distribute the Numb polarity protein asymmetrically and undergo hyper-proliferation, resulting in "cortical dysplasia." As disruption in cell polarity occurs in many forms of cancer, the loss of cell polarity in CNS progenitors may cause brain tumors in children (Klezovitch et al. 2004). 
The cerebellum: a model for neurogenesis, neurite outgrowth, and neuronal migration

The cerebellar cortex, or "little cortex," has long been an important model system for CNS development, owing to its near crystalline array of two principal classes of neurons, the granule cell and the Purkinje neuron. The Purkinje neuron, like the pyramidal cells of neocortex or hippocampus, is the sole output neuron of the cerebellum. Granule neurons form synaptic contacts with Purkinje cells, which provide the scaffolding for the primary circuitry of the cerebellar cortex. The output of the cerebellar circuitry depends on both connections of the granule cell with mossy fibers from a number of sources, and on connections of Purkinje cells with climbing fibers from the olivary nucleus, as well as local circuit synapses with a variety of cerebellar interneurons. Recent studies provide evidence that the cerebellum, once thought to function mainly in balance and motor control, is central to motor learning, sensory discrimination, and complex cognitive tasks. Thus, recent studies underscore the parallels between the structure and function of the cerebellum and the cerebrum.

The unique morphology of cerebellar granule neurons, extending two parallel fiber axons and multiple short, claw-like dendrites, develops in a series of steps that are faithfully recapitulated in vitro (Powell et al. 1997). The granule cell provides a remarkable model system, because it develops postnatally and because of the vast numbers of granule neurons, outnumbering the total population of neurons in the cerebral cortex. The latter allows purification of large numbers of granule cell progenitors for cell and molecular studies of neurogenesis, migration, and synaptogenesis. Progenitors of the granule neuron continue to divide until the end of the second postnatal week, facilitating studies on the molecular components of neuronal polarity and their function in neurite extension. A number of gene pathways promote cell division in granule cell progenitors at the expense of neuronal differentiation, including $\mathrm{N}-\mathrm{myc}$, sonic hedgehog, and Notch2 (Wechsler-Reya and Scott 1999; Kenney and Rowitch 2000; Solecki et al. 2001; Kenney et al. $2003,2004)$. Since granule cell progenitors proliferate well into the postnatal period, functional assays can be used to assess the contribution of candidate genes and/or pathways to neurogenesis and axon extension. The ability to perform functional bioassays, as well as monitor gene expression (e.g., RT-PCR) of large panels of genes, with an identified CNS neuron is a primary advantage of the cerebellar system.

\section{The centrosome governs axon initiation}

Recent studies point to an important role for the centrosome in granule cell differentiation. Centrosomes serve as the MTOC of most cell types, including neurons (Spiegelman et al. 1979). The centrosome coordinates a radial array of microtubules, anchoring the minus end of microtubules as the growing plus ends extend into the cell. While actin and intermediate filaments also grow in a polarized direction, they lack the tertiary organization provided by the centrosome. Proteomic studies indicate that centrosomes contain $>100$ structural proteins. Two signature structural proteins of the centrosome are Pericentrin and PCM-1, both of which recruit $\gamma$-tubulin to the tubulin ring complexes ( $\gamma$-tuRC). The critical role of the centrosome in axon extension was first proposed by Lefcort and Bentley (1989) in their classic analysis of axon genesis in grasshopper Til pioneer neurons. Although this model was thought to be restricted to invertebrates, studies in granule neurons (Zmuda and Rivas 1998 ) and, more recently, dissociated hippocampal neurons (de Anda et al. 2005) generalized the Lefcort and Bentley model to mammalian axons.

The development of granule neuron polarity can be examined in vitro as purified progenitor cells exit the cell cycle and develop the classic morphology of granule neurons in vivo (Powell et al. 1997). Within 12-20 h of plating, purified granule cell precursors exit the cell cycle. Subsequently, the centrosome moves to one pole of the cell from which a lamellipodium protrudes and develops into an axon. The Golgi and late/recycling endosomes also localize at the pole that generates the first neurite (Zmuda and Rivas 1998). The centrosome then relocates to the other side of the cell, and a second axon emerges, generating the classic bipolar morphology of immature granule neurons seen in vivo (Fig. 2). Disruption of actin polymerization blocks centrosome movement, and arrests granule cell differentiation at the unipolar phase (Zmuda and Rivas 2000), illustrating the critical role of cell polarity in axon outgrowth.

The two nascent granule cell axons, or parallel fibers, extend 50-75 $\mathrm{mm}$, prior to a third repositioning of the centrosome. The latter orients the granule cell soma perpendicular to the plane of axon extension and relocates the centrosome/MTOC adjacent to the site where a leading process emerges during the migration of the soma along Bergmann glia fibers (Fig. 2). As will be discussed in greater detail later in the review, the centrosome adopts a position at the base of the leading process during glial-guided neuronal migration, where it controls the timing of nuclear translocation and cell movement to the position where the granule cell will form dendritic arbors. Studies on purified cerebellar granule neurons therefore illustrate the key role of the centrosome, providing a compass for axon navigation and a chronometer for glial-guided migration during CNS development.

\section{Polarity proteins and signaling pathways in axon extension}

Recent studies show that Par proteins localize at the nascent tip of extending the extending axon, raising the possibility that polarity proteins drive the cytoskeletal motors that advance the growth cone. Extracellular cues have long been known to promote axon growth via specific receptors, G-proteins, and PAK-associated proteins. Recently, evidence has accumulated for a model involving deployment of the Par proteins to the tip of the growth cone. In this case, activated Cdc42 GTPase trig- 
1.

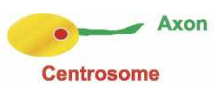

2.

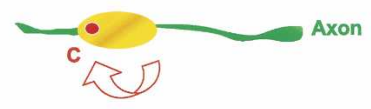

3.

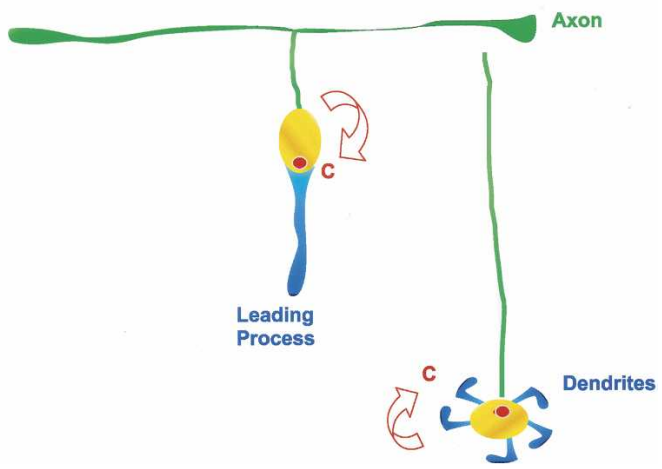

Figure 2. Centrosomal position during granule cell neurite extension, migration, and dendritic growth. Within $0.5 \mathrm{~h}$ of plating, cerebellar granule neurons extend short processes and orient the centrosome (red) to one side of the cell. (1) A process bearing a lamellopodia emerges from a site near the centrosome and develops into axon. (2) Thereafter, the centrosome relocates to the other side of the cell, where it organizes the outgrowth of a second axon, generating the characteristic bipolar shape of cerebellar granule neurons. As the axons extend rapidly, the centrosome moves to a perinuclear position and the cell soma begins to move along the radially aligned Bergmann glial fibers. (3) During migration, the centrosome is oriented in the direction of migration. (4) In post-mitotic granule neurons, short claw-like dendrites form synaptic connections with mossy fibers from nuclei in the brainstem. The leading process of the migrating neuron (blue) and the dendrites of the post-migratory granule cell (blue) express markers in common that distinguish them from the granule cell axon (green). For details, see Powell et al. (1997), Zmuda and Rivas (1998, 2000), and Solecki et al. (2004).

gers the recruitment of the mPar6 $\alpha$ polarity complexincluding Par3, Par6, and aPKC - to the membrane at the leading edge of the growth cone (Wiggin et al. 2005). Par1 (MARK) mediates the shuttling of the mPar6 $\alpha$ complex to and from the membrane. The localization of the mPar6 $\alpha$ complex involves the scaffold protein 14-3-3 (Par5), which binds to MARK2 in screens for proteinprotein interactions. It should be noted that kinases are often involved in multiple signaling cascades. For example, MARK isoforms regulate microtubule dynamics, the Par cell polarity determinants, and the Wnt- $\beta$ catenin signaling pathway (Sun et al. 2001).

This model for Cdc42 signaling mechanisms in axon extension is consistent with general mechanisms thought to polarize cell movements in response to extracellular cues. Classical studies on chemotaxic movements of slime mould and lymphocytes, as well as experiments on fibroblasts and neural crest cells, illustrate that Cdc 42 activity controls the polarization of the Golgi and centrosome in the direction of movement and restricts actin polymerization to the forward or leading edge, where lamellipodial protrusions emerge. In astro- cytes and fibroblasts, Cdc 42 activates the $\mathrm{mPar} 6 \alpha / \mathrm{PKC} \zeta$ complex, to polarize the centrosome and Golgi in the direction of lamellipodial formation and movement (Fig. 1). Cdc42 apparently regulates polarity of the centrosome through the mPar6 complex and actin/lamellipodia formation through $\beta$ Pix and Rac activation (EtienneManneville and Hall 2001, 2003; Cau and Hall 2005).

In hippocampal neurons, $\mathrm{Cdc} 42$ apparently activates the Par6 polarity complex during axon formation (Schwamborn and Puschel 2004) and elicits actin reorganization through the Par3 polarity protein interactions with Racspecific GEFs. Two Rac-specific guanine exchange factors (GEFs), Tiam1 (invasion-inducing T-lymphoma and metastasis 1) and STEF (SIF and Tiam1-like exchange factor), interact with Par3 (Chen and Macara 2005; Nishimura et al. 2005). Activated Cdc42 binds and activates the mPar6 $\alpha$ complex, after which Par3 interacts with the two exchange factors to localize the assembly at the tip of the axon. Thus, in CNS neurons, Cdc 42 activates the Par6/Par3 complex to coordinate centrosome positioning and actin dynamics required for axon formation.

The number of axons that particular neurons extend during development governs the connectivity of the synaptic circuit. Cerebellar granule cells extend two axons sequentially, as discussed above, except in circumstances where differentiation is blocked, when supernumerary fine processes are observed. Hippocampal cells, in contrast, appear to form many fine processes, one of which becomes the axon. At present, it is not clear whether these two classes of CNS neurons have different patterns of axon extension or whether live imaging in vivo is required to "capture" an earlier, transient stage of axon formation in hippocampal neurons. In hippocampal cells, the tubulin-binding protein CRMP-2 (collapsin response mediator protein-2) promotes the formation of supernumerary axons and increases axon branching (Inagaki et al. 2001; Fukata et al. 2002; Chen and Macara 2005; Nishimura et al. 2005) by binding to tubulin heterodimers and enhancing microtubule assembly in vitro. Importantly, the binding of a CRMP-2 mutant unable to induce microtubule assembly inhibits axonal growth and branching (Fukata et al. 2002). CRMP-2 also binds to Numb to regulate axon growth and endocytosis of the axonal guidance molecule L1 (Nishimura et al. 2003). L1 associates with the neuropilin-plexin receptor for semaphorin and modulates the effects of the semaphorins on guidance (Castellani et al. 2000, 2002). Thus CRMP-2, which mediates the effects of semaphorins on axon guidance (Goshimal et al. 1995), may also influence the response of growth cones to semaphorins by regulating L1 endocytosis. An important goal for the future will be to understand the coordinated response of the cytoskeleton to signaling cascades induced by guidance molecule-triggered receptor activation and polarity proteins at the tip of growing axons and dendrites.

\section{Polarity proteins in glial-guided CNS migration}

The migration of cerebellar granule cells along Bergmann glia in the cerebellar cortex provides a classical 
model for the migration of cortical neurons along the radial glia (Rakic 1971, 1972). During glial-guided migrations, immature CNS neurons express a highly polarized morphology and mode of movement. During migration, the Golgi, centrosome, and associated microtubule networks polarize the neuron in the direction of movement (Fig. 3; Edmondson and Hatten 1987; Gregory et al. 1988; Hatten 1999). Whereas the extending growth cone forms focal adhesions at the leading edge, migrating cells establish a broad interstitial junction beneath the cell soma (Gregory et al. 1988). In contrast to extending growth cones, the movement of the tip of the leading process does not correlate with the movement of the cell soma (Edmondson and Hatten 1987; Gregory et al. 1988), arguing that there is no "leading edge" at the tip of the leading process. Instead, the "motors" for glial-guided migration appear to be localized in the cell soma.

The similarity in the mode of cell movement of cerebellar, cortical neurons, and/or hippocampal neurons

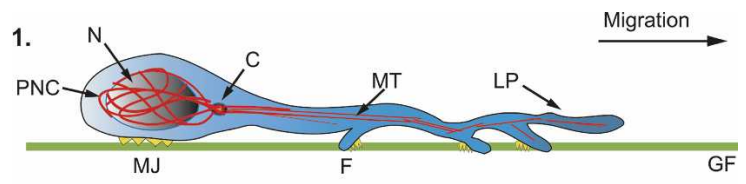

2.

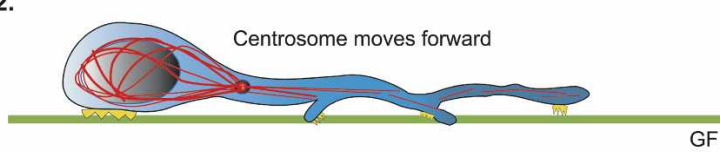

3.

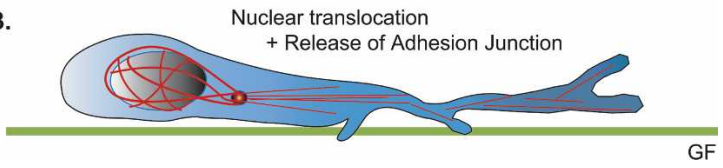

Figure 3. During glial-guided of migration of CNS neurons in the developing mammalian brain, the centrosome controls the polarity and direction of cell movement. (1) The neuron is polarized in the direction of migration (arrow) with the nucleus $(\mathrm{N})$ in the posterior aspect of the cell. The nucleus, Golgi apparatus (not shown) and centrosome $(\mathrm{C}, \mathrm{red})$ are forward of the nucleus, and a system of microtubules (MT, red) extends into the leading process from the MTOC. An interstitial junction forms beneath the cell soma (MJ, yellow) and punctae adherentia (yellow) form beneath the short $(1-5 \mu \mathrm{m})$ filopodia $(\mathrm{F})$, extending from the leading process as it enwraps the glial fiber (GF, green). In contrast to growth cones, there is no "leading edge" at the tip of the leading process, and although the leading process is highly motile, the movement of the cell corresponds to the movement of the cell soma, not the movement of the tip of the leading process. (2) The centrosome moves forward, leading to stretching of the perinuclear tubulin cage surrounding the nucleus. (3) As the interstitial junction dissipates, the nucleus and soma move forward, leading to additional deformation of the perinuclear tubulin cage and swelling of the proximal area of the leading process. The neuron moves along the glial fiber -1.5-2.0 $\mu \mathrm{m}$, after which a new interstitial junction anchors the cell soma. Migration continues in a saltatory manner, as the cycle of forward movement of the centrosome followed by release of the interstitial junction and somal translocation repeats. along glial fibers suggested a common mechanism of glial-guided migration for the large output neurons of neocortex, pyramidal cells of hippocampus and principal neurons of the cerebellar cortex (Rakic 1990, 2000; Hatten 1999, 2002). Migrating neurons express unique cytoskeletal features, including a perinuclear "cage" of microtubules and a thick band of cortical actin that lines the soma. Gene expression profiling of mitotic and postmitotic granule cell precursors (D.J. Solecki and M.E. Hatten, unpubl.) reveal expression of several classes of conserved polarity proteins, including the mPar $6 \alpha$ complex, in post-mitotic granule neurons, just prior to differentiation. To examine the role of polarity proteins in neuronal migration, Solecki et al. (2004) imaged the conserved polarity complex mPar6 $\alpha$ in migrating and stationary cerebellar granule neurons. As the neuron migrates, Par $6 \alpha / \mathrm{PKC} \zeta$ localizes to the centrosome and signals forward movement of the centrosome prior to the translocation of the cell soma. Thus, migration occurs by a "two-stroke" mechanism controlled by mPar6 $\alpha$ signaling (Fig. 3; Solecki et al. 2004). Overexpression of mPar6 $\alpha$ disrupts the stoichiometry of polarity complexes and inhibits glial-guided migration. The perinuclear tubulin cage seen in migrating neurons is absent, and a number of "signature" centrosome proteins, including $\gamma$-tubulin, Pericentrin, centrin2, PKC $\zeta$ and $\mathrm{mPar} 6 \alpha$, fail to target to the centrosome. Most importantly, the remaining centrosomal assembly stops moving, suggesting that mPar $6 \alpha$ signaling regulates centrosome motility (Solecki et al. 2004). Recent studies on spindle poles and interphase centrosomes suggest that some pericentriolar matrix (PCM) components, including $\gamma$-tubulin, are transported to centrosomes via dynein/ dynactin (Young et al. 2000; Goshima et al. 2005). Since a variety of important PCM components that are dynein cargoes fail to target to the centrosome in the absence of mPar6 $\alpha$, mPar6 $\alpha$ signaling might also regulate dynein/ dynactin activity. Deciphering the signaling pathways that regulate the activity of the mPar6 $\alpha$ polarity complex as well as the downstream control of cytoskeletal dynamics, contractile force generation, and vesicle trafficking will hopefully lead to insights on the defects in formation of the cortical laminae that characterize human brain malformations and many of the epilepsies (for review, see Ross and Walsh 2001).

\section{Polarity proteins and pathways in synaptogenesis}

Once the axon has reached its target, a series of changes in neuronal polarity occur to induce presynaptic and post-synaptic specializations. Signaling cascades coordinate cytoskeletal dynamics and regulate endocytosis and protein trafficking dynamics required for the synaptic circuitry to form and function. The formation of pre- and post-synaptic specializations follows the general mechanism, described earlier, for the formation of intercellular junctions in mammalian epithelia: Adhesion ligands mediate initial contacts that then stabilize as polarity proteins and cytoskeletal elements localize to the junctional site. In Drosophila, as shown by Goodman and 
colleagues (Grenningloh et al. 1991), guidepost cell fasciclin II regulates the pattern of synapse formation; in $C$. elegans, as shown by Bargmann and colleagues (Shen and Bargmann 2003; Shen et al. 2004), the synaptic guidepost protein SYG-2 and its receptor, SYG-1, generate synaptic specificity. In mouse hippocampal cells, SynCAM 1 (Synaptic Cell Adhesion Molecule 1) and neuroligin trigger presynaptic development in contacting axons and pattern synaptic assembly (Schieffle et al. 2000; Biederer and Sudhof 2001; Biederer et al. 2002; Sara et al. 2005). Recent experiments (Kishi et al. 2005; Inoue et al. 2006) demonstrated that SAD-A and SAD-B, mammalian orthologs of a kinase needed for presynaptic differentiation in C. elegans, function in neuronal polarization during synapse formation in vertebrates, again establishing the conservation of molecular mechanisms between species.

Studies on neuronal differentiation of the cerebellar granule cell provided the first indication showing that axon outgrowth involves the development of synaptic vesicle trafficking, in addition to the development of cytoskeletal dynamics. The conserved serine-threonine kinase unc-51 binds the synaptic protein syngap, the scaffolding protein Syntenin and rab5 as an early step in the development of synaptic vesicle trafficking. Genetic studies in C. elegans and binding studies in the mouse cerebellum show that unc-51 (Ulk1) phosphorylates the Vab-8 protein, a kinesin domain-containing protein that functions in posterior cell migration and axon guidance in C. elegans (Lai and Garriga 2004). Recent studies in Drosophila show that Syntenin and rab5 are core components of the vesicle trafficking machinery, as loss of syntaxin (avalanche) results in accumulation of the polarity proteins Notch and Crumbs from a failure in endosomal entry and progression toward lysosomal degradation. These findings suggest that the establishment of synaptic vesicle trafficking may regulate neuronal polarity by controlling the localization of polarity proteins within axons, dendrites, and synapses.

Sequestration of proteins within the growth cone and nascent synapses is likely accomplished by a combination of active transport and tethering to the actin cytoskeleton. Measurement of the lateral mobility of membrane proteins at the tip of growing axons indicates that some proteins move very slowly in the initial segment of the axon. This apparent "diffusion barrier" is abolished by agents that disrupt actin filaments, suggesting that polarity proteins are maintained at the growing tip, at least in part, by differential tethering to the cytoskeleton (Nakada et al. 2003). The diffusion barrier at the tip of axons and dendrites generates a high density of proteins, apparently arranged in rows. The dense membrane/cytoskeleton/protein array even stops the rapid diffusion of phospholipids, which may provide a model for the formation of diffusion barriers in epithelial membranes. The classic work of Scheller (Steegmaier et al. 1998; Chao et al. 1999) shows that evolutionarily conserved mechanisms of protein transport across the membrane and intracellular trafficking are part of the process that generates and maintains neuronal polarity at synapses in the developing and mature brain. The present challenge is to integrate the role of polarity proteins and modes of intracellular transport, especially within the context of axon guidance and the maturation of particular synapses, providing a new and potentially important approach to understanding cognitive functions in the mammalian brain.

\section{Polarity of mRNA localization in CNS synapses}

In the hippocampus, as in other brain regions, axons form presynaptic contacts with the dendrites of appropriate targets. Two decades ago, Davis and Steward (Davis et al. 1987) discovered polyribosomes beneath the post-synaptic specializations on dendrites of cultured hippocampal neurons. Steward (Steward and Wallace 1995) went on to show that the distribution of mRNAs in the hippocampus correlated with afferent innervation patterns. By in situ analysis, they observed a laminar pattern of expression that recapitulated the pattern of innervation of hippocampal neurons (dendrites) by the two major axonal projections into the hippocampus. Thus, experiments on cultured hippocampal neurons suggested that the localization of translation machinery and

mRNAs at synapses enables those synapses to independently control synaptic strength through the local synthesis of proteins (for review, see Martin et al. 2000; Steward and Schuman 2001). To identify proteins that regulate mRNA targeting to specific post-synaptic junctions, a number of laboratories have studied mammalian genes related to the Drosophila gene Staufen, because the Staufen protein localizes mRNAs to specific cellular domains (Fuerstenberg et al. 1998; Shen et al. 1998; Roegiers and Jan 2000). Two mammalian staufen genes encode proteins containing conserved double-stranded mRNA-binding domains expressed in hippocampal neurons. An interesting feature of Staufen proteins is the formation of granules that colocalize with ribonuclear particles, which transport mRNA to the dendrites (Tang et al. 2001). In the absence of Stau2, the number of dendritic spines decreases and filopodia-like structures increase, suggesting that Stau2 RNA-binding protein functions in mRNA trafficking in hippocampal dendrites (Kiebler et al. 1999; Kohrmann et al. 1999; Macchi et al. 2003; Goetze et al. 2006). The role of polarity complex proteins in synaptic plasticity, a specialized form of mammalian epithelial cell junctions, is a central unanswered question.

As storage of long-term memory requires protein synthesis, the deployment of polyribosomes to dendrites appears to modulate long-term potentiation of synaptic activity (L-LTP) and long-term depression of synaptic activity (L-LTD). Transgenic mice expressing a dominantnegative form of MEK1 that inactivates the mitogen-acitvated protein kinase (MAPK) pathway have selective defects in L-LTP, suggesting that MAPK signaling modifies gene expression involved in synaptic plasticity. In cultured hippocampal cells, inhibition of MAPK signaling blocks activity-induced translation and phosphorylation of translation factors eIF4E and 4EBP1 and ribo- 
somal protein S6 in post-synaptic regions of cultured hippocampal neurons, suggesting that neuronal activity modulates local, synaptic mRNA translation (Kelleher et al. 2004a,b).

As Par proteins regulate both protein and mRNA transport, they are likely to function in the maintenance of trafficking required for dendritic function in mammals. In Drosophila, a protein related to the human Fragile $\mathrm{X}$ mental retardation protein (FMRP), binds mRNAs and the polarity protein Lgl. Subsequently, the Par protein assembly localizes the FMRP/mRNA/Lgl complex to synapses (Zarnescu et al. 2005). As many functions of Par polarity proteins are conserved across species, results from studies in Drosophila suggest that Par proteins may target specific mRNAs to synapses and thereby modulate synaptic strength. As human FMRP is widely assumed to function in human cognition, it will be very important to examine whether the Par proteins localize FMRP and/or other mRNAs/proteins to synapses related to higher mental functions. The role of polarity proteins in molecular pathways that set synaptic activity is a key question in unraveling mechanisms that control the functions of synaptic circuits in brain. This general question relates to thee pressing problem as to whether the local synthesis of particular proteins sets the activity level of key synapses in cortical circuits? Second, do mammalian polarity proteins interact with FMRP and help regulate translation of specific sets of dendritic RNA (Darnell et al. 2005) at critical synapses in the cortical circuitry?

The discovery that evolutionarily conserved proteins control neuronal polarity has been a surprising development that will likely propel progress on basic problems in developmental neurobiology at an ever-increasing pace. All fundamental steps in CNS development-including neurogenesis in specialized neuroepithelia, coordination of the centrosome and neurite outgrowth, the formation of a specialized adhesion during neuronal migration, and the formation of synaptic contacts that are highly specialized epithelial junctions-are likely to involve polarity proteins. Thus, understanding the molecular processes governed by these proteins is likely to provide new insights on the development of brain circuitry and cognitive function.

\section{Acknowledgments}

We are especially grateful to Dr. Kathryn Zimmerman for critically reading the manuscript. This work was supported by NIHNS R01 15429-28 (to M.E.H.).

\section{References}

Anthony, T.E., Klein, C., Fishell, G., and Heintz, N. 2004. Radial glia serve as neuronal progenitors in all regions of the central nervous system. Neuron 41: 881-890.

Biederer, T. and Sudhof, T.C. 2001. CASK and protein 4.1 support F-actin nucleation on neurexins. J. Biol. Chem. 276: 47869-47876.

Biederer, T., Sara, Y., Mozhayeva, M., Atasoy, D., Liu, X., Kavalali, E.T., and Sudhof, T.C. 2002. SynCAM, a synaptic ad- hesion molecule that drives synapse assembly. Science 297: 1525-1531.

Castellani, V., Chedotal, A., Schachner, M., Faivre-Sarrailh, C., and Rougon, G. 2000. Analysis of the L1-deficient mouse phenotype reveals cross-talk between Sema3A and L1 signaling pathways in axonal guidance. Neuron 27: 237-249.

Castellani, V., De Angelis, E., Kenwrick, S., and Rougon, G. 2002. Cis and trans interactions of $\mathrm{L} 1$ with neuropilin-1 control axonal responses to semaphorin 3A. EMBO I. 21: 63486357.

Cau, J. and Hall, A. 2005. Cdc42 controls the polarity of the actin and microtubule cytoskeletons through two distinct signal transduction pathways. J. Cell Sci. 118: 2579-2587.

Chao, D.S., Hay, J.C., Winnick, S., Prekeris, R., Klumperman, J., and Scheller, R.H. 1999. SNARE membrane trafficking dynamics in vivo. J. Cell Biol. 144: 869-881.

Chen, X. and Macara, I.G. 2005. Par-3 controls tight junction assembly through the Rac exchange factor Tiam1. Nat. Cell Biol. 7: 262-269.

Darnell, J.C., Mostovetsky, O., and Darnell, R.B. 2005. FMRP RNA targets: Identification and validation. Genes Brain Behav. 4: 341-349.

Davis, L., Banker, G.A., and Steward, O. 1987. Selective dendritic transport of RNA in hippocampal neurons in culture. Nature 330: 477-479.

de Anda, F.C., Pollarolo, G., Da Silva, J.S., Camoletto, P.G., Feiguin, F., and Dotti, C.G. 2005. Centrosome localization determines neuronal polarity. Nature 436: 704-708.

Doe, C.Q. and Bowerman, B. 2001. Asymmetric cell division: Fly neuroblast meets worm zygote. Curr. Opin. Cell Biol. 13: 68-75.

Edmondson, J.C. and Hatten, M.E. 1987. Glial-guided granule neuron migration in vitro: A high-resolution time-lapse video microscopic study. J. Neurosci. 7: 1928-1934.

Etienne-Manneville, S. and Hall, A. 2001. Integrin-mediated activation of $\mathrm{Cdc} 42$ controls cell polarity in migrating astrocytes through PKC $\zeta$. Cell 106: 489-498.

- 2003. Cell polarity: Par6, aPKC and cytoskeletal crosstalk. Curr. Opin. Cell Biol. 15: 67-72.

Faulkner, N.E., Dujardin, D.L., Tai, C.Y., Vaughan, K.T., O'Connell, C.B., Wang, Y., and Vallee, R.B. 2000. A role for the lissencephaly gene LIS1 in mitosis and cytoplasmic dynein function. Nat. Cell Biol. 2: 784-791.

Fuerstenberg, S., Broadus, J., and Doe, C.Q. 1998. Asymmetry and cell fate in the Drosophila embryonic CNS. Int. J. Dev. Biol. 42: 379-383.

Fukata, M., Nakagawa, M., Kuroda, S., and Kaibuchi, K. 2002. Effects of Rho family GTPases on cell-cell adhesion. Methods Mol. Biol. 189: 121-128.

Gao, L. and Macara, I.G. 2004. Isoforms of the polarity protein par6 have distinct functions. J. Biol. Chem. 279: 4155741562.

Gleeson, J.G. 2001. Neuronal migration disorders. Ment. Retard. Dev. Disabil. Res. Rev. 7: 167-171.

Gleeson, J.G. and Walsh, C.A. 2000. Neuronal migration disorders: From genetic diseases to developmental mechanisms. Trends Neurosci. 23: 352-359.

Goetze, B., Tuebing, F., Xie, Y., Dorostkar, M.M., Thomas, S., Pehl, U., Boehm, S., Macchi, P., and Kiebler, M.A. 2006. The brain-specific double-stranded RNA-binding protein Staufen 2 is required for dendritic spine morphogenesis. $J$. Cell Biol. 172: 221-231.

Goshima, Y., Nakamura, F., Strittmatter, P., and Strittmatter, S.M. 1995. Collapsin-induced growth cone collapse mediated by an intracellular protein related to UNC-33. Nature 376: 509-514. 
Goshima, G., Nedelec, F., and Vale, R.D. 2005. Mechanisms for focusing mitotic spindle poles by minus end-directed motor proteins. J. Cell Biol. 171: 229-240.

Gregory, W., Edmondson, J., Hatten, M., and Mason, C. 1988. Cytology and neuron-glial apposition of migrating cerebellar granule cells in vitro. J. Neurosci. 8: 1728-1738.

Grenningloh, G., Rehm, E.J., and Goodman, C.S. 1991. Genetic analysis of growth cone guidance in Drosophila: Fasciclin II functions as a neuronal recognition molecule. Cell 67: 45-57.

Hatten, M.E. 1999. Central nervous system neuronal migration. Annu. Rev. Neurosci. 22: 511-539.

. 2002. New directions in neuronal migration. Science 297: $1660-1663$.

2005. LIS-less neurons don't even make it to the starting gate. J. Cell Biol. 170: 867-871.

Inagaki, N., Chihara, K., Arimura, N., Menager, C., Kawano, Y., Matsuo, N., Nishimura, T., Amano, M., and Kaibuchi, K. 2001. CRMP-2 induces axons in cultured hippocampal neurons. Nat. Neurosci. 4: 781-782.

Kelleher III, R.J., Govindarajan, A., Jung, H.Y., Kang, H., and Tonegawa, S. 2004a. Translational control by MAPK signaling in long-term synaptic plasticity and memory. Cell 116: 467-479.

Kelleher III, R.J., Govindarajan, A., and Tonegawa, S. 2004b. Translational regulatory mechanisms in persistent forms of synaptic plasticity. Neuron 44: 59-73.

Kenney, A.M. and Rowitch, D.H. 2000. Sonic hedgehog promotes G(1) cyclin expression and sustained cell cycle progression in mammalian neuronal precursors. Mol. Cell. Biol. 20: 9055-9067.

Kenney, A.M., Cole, M.D., and Rowitch, D.H. 2003. Nmyc upregulation by sonic hedgehog signaling promotes proliferation in developing cerebellar granule neuron precursors. Development 130: 15-28.

Kenney, A.M., Widlund, H.R., and Rowitch, D.H. 2004. Hedgehog and PI-3 kinase signaling converge on Nmycl to promote cell cycle progression in cerebellar neuronal precursors. Development 131: 217-228.

Kiebler, M.A., Hemraj, I., Verkade, P., Kohrmann, M., Fortes, P., Marion, R.M., Ortin, J., and Dotti, C.G. 1999. The mammalian staufen protein localizes to the somatodendritic domain of cultured hippocampal neurons: Implications for its involvement in mRNA transport. J. Neurosci. 19: 288-297.

Kim, S.K. 2000. Cell polarity: New PARtners for Cdc42 and Rac. Nat. Cell Biol. 2: E143-E145.

Kishi, M., Pan, Y.A., Crump, J.G., and Sanes, J.R. 2005. Mammalian SAD kinases are required for neuronal polarization. Science 307: 929-932.

Klezovitch, O., Fernandez, T.E., Tapscott, S.J., and Vasioukhin, V. 2004. Loss of cell polarity causes severe brain dysplasia in Lgl1 knockout mice. Genes \& Dev. 18: 559-571.

Kohrmann, M., Luo, M., Kaether, C., DesGroseillers, L., Dotti, C.G., and Kiebler, M.A. 1999. Microtubule-dependent recruitment of Staufen-green fluorescent protein into large RNA-containing granules and subsequent dendritic transport in living hippocampal neurons. Mol. Biol. Cell 10: 29452953.

Lai, T. and Garriga, G. 2004. The conserved kinase UNC-51 acts with VAB-8 and UNC-14 to regulate axon outgrowth in $C$. elegans. Development 131: 5991-6000.

Langevin, J., Le Borgne, R., Rosenfeld, F., Gho, M., Schweisguth, F., and Bellaiche, Y. 2005. Lethal giant larvae controls the localization of notch-signaling regulators numb, neuralized, and Sanpodo in Drosophila sensory-organ precursor cells. Curr. Biol. 1: 955-962.

Lefcort, F. and Bentley, D. 1989. Organization of cytoskeletal elements and organelles preceding growth cone emergence from an identified neuron in situ. J. Cell Biol. 108: 17371749.

Macara, I.G. 2004. Par proteins: Partners in polarization. Curr. Biol. 14: R160-R162.

Macchi, P., Hemraj, I., Goetze, B., Grunewald, B., Mallardo, M., and Kiebler, M.A. 2003. A GFP-based system to uncouple mRNA transport from translation in a single living neuron. Mol. Biol. Cell 14: 1570-1582.

Martin, K.C., Barad, M., and Kandel, E.R. 2000. Local protein synthesis and its role in synapse-specific plasticity. Curr. Opin. Neurobiol. 10: 587-592.

Nakada, C., Ritchie, K., Oba, Y., Nakamura, M., Hotta, Y., Iino, R., Kasai, R.S., Yamaguchi, K., Fujiwara, T., and Kusumi, A. 2003. Accumulation of anchored proteins forms membrane diffusion barriers during neuronal polarization. Nat. Cell Biol. 5: 626-632.

Nishimura, T., Fukata, Y., Kato, K., Yamaguchi, T., Matsuura, Y., Kamiguchi, H., and Kaibuchi, K. 2003. CRMP-2 regulates polarized Numb-mediated endocytosis for axon growth. Nat. Cell Biol. 5: 819-826.

Nishimura, T., Yamaguchi, T., Kato, K., Yoshizawa, M., Nabeshima, Y., Ohno, S., Hoshino, M., and Kaibuchi, K. 2005. PAR-6-PAR-3 mediates Cdc42-induced Rac activation through the Rac GEFs STEF/Tiam1. Nat. Cell Biol. 7: 270277.

Noctor, S.C., Martinez-Cerdeno, V., Ivic, L., and Kriegstein, A.R. 2004. Cortical neurons arise in symmetric and asymmetric division zones and migrate through specific phases. Nat. Neurosci. 7: 136-144.

O'Connell, C.B. and Wang, Y.L. 2000. Mammalian spindle orientation and position respond to changes in cell shape in a dynein-dependent fashion. Mol. Biol. Cell 11: 1765-1774.

Pellettieri, J. and Seydoux, G. 2002. Anterior-posterior polarity in C. elegans and Drosophila-PARallels and differences. Science 298: 2981946-2981950.

Petersen, P.H., Zou, K., Hwang, J.K., Jan, Y.N., and Zhong, W. 2002. Progenitor cell maintenance requires numb and numblike during mouse neurogenesis. Nature 419: 929-934.

Petersen, P.H., Zou, K., Krauss, S., and Zhong, W. 2004. Continuing role for mouse $\mathrm{Numb}$ and $\mathrm{Numbl}$ in maintaining progenitor cells during cortical neurogenesis. Nat. Neurosci. 7: 803-811.

Powell, S.K., Rivas, R.J., Rodriguez-Boulan, E., and Hatten, M.E. 1997. Development of polarity in cerebellar granule neurons. J. Neurobiol. 32: 223-236.

Rakic, P. 1971. Neuron-glia relationship during granule cell migration in developing cerebellar cortex. A Golgi and electronmicroscopic study in Macacus Rhesus. J. Comp. Neurol. 141: 283-312.

-1972. Mode of cell migration to the superficial layers of fetal monkey neocortex. J. Comp. Neurol. 145: 61-83.

1990. Principles of neural cell migration. Experientia 46: 882-891.

- 2000. Molecular and cellular mechanisms of neuronal migration: Relevance to cortical epilepsies. Adv. Neurol. 84: $1-14$.

Roegiers, F. and Jan, Y.N. 2000. Staufen: A common component of mRNA transport in oocytes and neurons? Trends Cell Biol. 10: 220-224.

Ross, M.E. and Walsh, C.A. 2001. Human brain malformations and their lessons for neuronal migration. Annu. Rev. Neurosci. 24: 1041-1070.

Sara, Y., Biederer, T., Atasoy, D., Chubykin, A., Mozhayeva, M.G., Sudhof, T.C., and Kavalali, E.T. 2005. Selective capability of SynCAM and neuroligin for functional synapse as- 
sembly. J. Neurosci. 25: 260-270.

Sauer, M.E. and Chittenden, A.C. 1959. Deoxyribonucleic acid content of cell nuclei in the neural tube of the chick embryo: Evidence for intermitotic migration of nuclei. Exp. Cell Res. 16: $1-6$.

Schieffle, P., Fan, J., Choih, J., Fetter, R., and Serafine, T. 2000. Neruoligin expressed in nonneuronal cells triggers presynaptic development in contacting acons. Cell 101: 657-669.

Schwamborn, J.C. and Puschel, A.W. 2004. The sequential activity of the GTPases Rap1B and Cdc42 determines neuronal polarity. Nat. Neurosci. 7: 923-929.

Shen, K. and Bargmann, C.I. 2003. The immunoglobulin superfamily protein SYG-1 determines the location of specific synapses in C. elegans. Cell 112: 619-630.

Shen, C.P., Knoblich, J.A., Chan, Y.M., Jiang, M.M., Jan, L.Y., and Jan, Y.N. 1998. Miranda as a multidomain adapter linking apically localized Inscuteable and basally localized Staufen and Prospero during asymmetric cell division in Drosophila. Genes \& Dev. 12: 1837-1846.

Shen, K., Fetter, R.D., and Bargmann, C.I. 2004. Synaptic specificity is generated by the synaptic guidepost protein SYG-2 and its receptor, SYG-1. Cell 116: 869-881.

Siller, K.H., Serr, M., Steward, R., Hays, T.S., and Doe, C.Q. 2005. Live imaging of Drosophila brain neuroblasts reveals a role for Lis1/dynactin in spindle assembly and mitotic checkpoint control. Mol. Biol. Cell 16: 5127-5140.

Solecki, D.J., Liu, X.L., Tomoda, T., Fang, Y., and Hatten, M.E. 2001. Activated Notch2 signaling inhibits differentiation of cerebellar granule neuron precursors by maintaining proliferation. Neuron 31: 557-568.

Solecki, D.J., Model, L., Gaetz, J., Kapoor, T.M., and Hatten, M.E. 2004. Par6 $\alpha$ signaling controls glial-guided neuronal migration. Nat. Neurosci. 7: 1195-1203.

Spiegelman, B.M., Lopata, M.A., and Kirschner, M.W. 1979. Aggregation of microtubule initiation sites preceding neurite outgrowth in mouse neuroblastoma cells. Cell 16: 253-263.

Steegmaier, M., Yang, B., Yoo, J.S., Huang, B., Shen, M., Yu, S., Luo, Y., and Scheller, R.H. 1998. Three novel proteins of the syntaxin/SNAP-25 family. J. Biol. Chem. 273: 34171-34179.

Steward, O. and Schuman, E.M. 2001. Protein synthesis at synaptic sites on dendrites. Annu. Rev. Neurosci. 24: 299-325.

Steward, O. and Wallace, C.S. 1995. mRNA distribution within dendrites: Relationship to afferent innervation. J. Neurobiol. 26: 447-449.

Sun, T.Q., Lu, B., Feng, J.J., Reinhard, C., Jan, Y.N., Fantl, W.J., and Williams, L.T. 2001. PAR-1 is a Dishevelled-associated kinase and a positive regulator of Wnt signalling. Nat. Cell Biol. 3: 628-636.

Sun, Y., Goderie, S.K., and Temple, S. 2005. Asymmetric distribution of EGFR receptor during mitosis generates diverse CNS progenitor cells. Neuron 45: 873-886.

Tang, S.J., Meulemans, D., Vazquez, L., Colaco, N., and Schuman, E. 2001. A role for a rat homolog of staufen in the transport of RNA to neuronal dendrites. Neuron 32: 463475.

Torban, E., Kor, C., and Gros, P. 2004. Van Gogh-like2 (Strabismus) and its role in planar cell polarity and convergent extension in vertebrates. Trends Genet. 20: 570-577.

Tsai, J.W., Chen, Y., Kriegstein, A.R., and Vallee, R.B. 2005. LIS1 RNA interference blocks neural stem cell division, morphogenesis, and motility at multiple stages. J. Cell Biol. 170: 935-945.

Wechsler-Reya, R.J. and Scott, M.P. 1999. Control of neuronal precursor proliferation in the cerebellum by Sonic Hedgehog. Neuron 22: 103-114.

Wiggin, G.R., Fawcett, J.P., and Pawson, T. 2005. Polarity pro- teins in axon specification and synaptogenesis. Dev. Cell 8: 803-816.

Wodarz, A., Ramrath, A., Grimm, A., and Knust, E. 2000. Drosophila atypical protein kinase $\mathrm{C}$ associates with Bazooka and controls polarity of epithelia and neuroblasts. I. Cell Biol. 150: 1361-1374.

Young, A., Dictenberg, J.B., Purohit, A., Tuft, R., and Doxsey, S.J. 2000. Cytoplasmic dynein-mediated assembly of pericentrin and $\gamma$ tubulin onto centrosomes. Mol. Biol. Cell 11: 2047-2056

Yu, F., Kuo, C.T., and Jan, Y.N. 2006. Drosophila neuroblast asymmetric cell division: Recent advances and implications for stem cell biology. Neuron 51: 13-20.

Zarnescu, D.C., Jin, P., Betschinger, J., Nakamoto, M., Wang, Y., Dockendorff, T.C., Feng, Y., Jongens, T.A., Sisson, J.C., Knoblich, J.A., et al. 2005. Fragile X protein functions with $\mathrm{lgl}$ and the par complex in flies and mice. Dev. Cell 8: 43-52.

Zmuda, J.F. and Rivas, R. 1998. The Golgi apparatus and the centrosome are localized to the sites of newly emerging axons in cerebellar neurons in vitro. Cell Motil. Cytoske1eton 41: 18-38.

. 2000. Actin disruption alters the localization of tau in the growth cones of cerebellar granule neurons. I. Cell Sci. 113: $2797-2809$ 


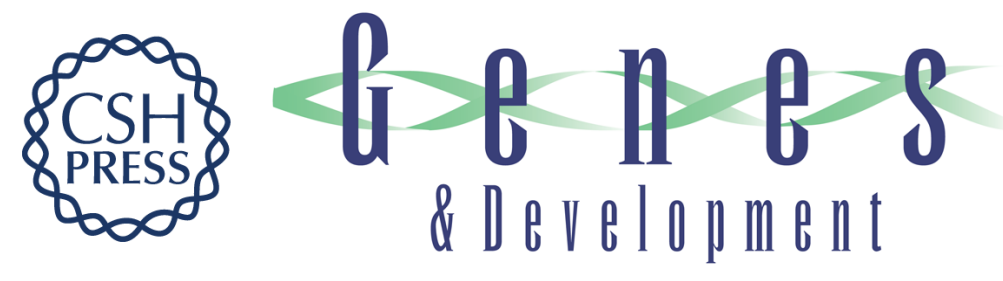

\section{Neuronal polarity in CNS development}

David J. Solecki, Eve-Ellen Govek, Toshifumi Tomoda, et al.

Genes Dev. 2006, 20:

Access the most recent version at doi:10.1101/gad.1462506

References This article cites 88 articles, 31 of which can be accessed free at: http://genesdev.cshlp.org/content/20/19/2639.full.html\#ref-list-1

License

Email Alerting Receive free email alerts when new articles cite this article - sign up in the box at the top Service right corner of the article or click here.

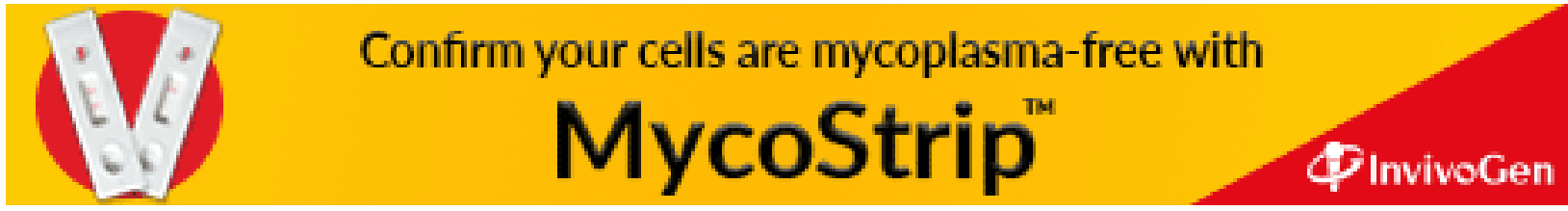

